\title{
Improved delivery of the OVA-CD4 peptide to $T$ helper cells by polymeric surface display on Salmonella
}

Junjie Zhang ${ }^{1,2,3}$, Leon De Masi ${ }^{2}$, Beena John², Wenxin Chen ${ }^{1}$ and Dieter M Schifferli ${ }^{2 *}$

\begin{abstract}
Background: Autotransporter proteins represent a treasure trove for molecular engineers who modify Gram-negative bacteria for the export or secretion of foreign proteins across two membrane barriers. A particularly promising direction is the development of autotransporters as antigen display or secretion systems. Immunologists have been using ovalbumin as a reporter antigen for years and have developed sophisticated tools to detect specific T cells that respond to ovalbumin. Although ovalbumin-expressing bacteria are being used to trace T cell responses to colonizing or invading pathogens, current constructs for ovalbumin presentation have not been optimized.
\end{abstract}

Results: The activation of T helper cells in response to ovalbumin was improved by displaying the OVA-CD4 reporter epitope as a multimer on the surface of Salmonella and fused to the autotransporter MisL. Expression was optimized by including tandem in vivo promoters and two post-segregational killing systems for plasmid stabilization.

Conclusions: The use of an autotransporter protein to present relevant epitope repeats on the surface of bacteria, combined with additional techniques favoring stable and efficient in vivo transcription, optimizes antigen presentation to $T$ cells. The technique of multimeric epitope surface display should also benefit the development of new Salmonella or other enterobacterial vaccines.

Keywords: Autotransporter, T5SS, Salmonella, MisL, Ovalbumin

\section{Background}

Escherichia coli is the prototype bacterium used for the production of desired foreign proteins in vitro. A particularly interesting approach has been the export of foreign proteins on the bacterial surface of $E$. coli by taking advantage of the specific inherent properties of autotransporter proteins. These proteins, which represent a category of type $\mathrm{V}$ secretion systems of Gram-negative bacteria, are exported to the periplasm by the sec machinery and assembled into the outer membrane by the Bam and Tam proteins [1-3]. The carboxy-terminal end of an autotransporter protein forms a beta-barrel structure with a central pore originally thought to channel the amino-terminal side of the protein or passenger

\footnotetext{
* Correspondence: dmschiff@vet.upenn.edu

${ }^{2}$ Department of Pathobiology, University of Pennsylvania School of Veterinary Medicine, Philadelphia, Pennsylvania 19104, USA

Full list of author information is available at the end of the article
}

domain to the bacterial surface. Newer models propose that Bam participates in making the pore, implying that the term "autotransporter" is a misnomer [3]. For some autotransporter proteins, the translocated segment is cleaved and released from the bacteria, which can be useful for direct purification procedures from spent medium. Some cleaved translocators remain surface associated by non-covalent bond. Whether secreted or on the bacterial surface, translocators can act as adhesins, mediators of biofilm formation, enzymes for intercellular spreading, cytotoxins or modulators of immune responses [3]. Early studies recognized the use of autotransporters as export machineries for foreign proteins and antigens. Translocator domains of a variety of autotransporter proteins have been modified with in-frame fusions of recombinant proteins for display on the bacterial surface or for delivery to the spent medium $[4,5]$. Among these autotransporters, the Salmonella 
MisL autotransporter adhesin [6] has previously been engineered to express four copies of the Plasmodium falciparum immunodominant epitope (NANP) on the surface of Salmonella enterica [7]. MisL was able to express even more copies of NANP on E. coli and to release them in the medium after the addition of a cleavage site for the surface protease OmpT [8]. The epitopes were shown to induce immune responses. Similarly, viral and parasitic protein epitopes were successfully expressed by recombinant MisL on Salmonella vaccine strains and induced epitope-specific immune responses with protective properties $[9,10]$.

Here, we took advantage of the transport property of MisL to export a fused antigenic model peptide on the surface of Salmonella as an activation signal for specific cellular immune responses. The generation of a $\mathrm{T}$ helper cell 1 (Th1) immune response is crucial for successful control of the facultative intracellular pathogen Salmonella and several studies have highlighted the importance of the CD4+ $\mathrm{T}$ cell response during infection [11]. The ability to track antigen specific $\mathrm{T}$ cells is important for understanding the initiation and maintenance of $\mathrm{T}$ cell responses during various infections and in response to vaccines [12]. Since endogenous $T$ cells specific for any given antigen are present in small numbers, it makes the tracking of such cells difficult during the early phases of an immune response before clonal expansion has occurred. Adoptive transfer models using TCR-transgenic $\mathrm{T}$ cells specific for model antigens such as ovalbumin have thus provided a vital tool for tracking antigen specific $\mathrm{T}$ cell responses [13-15]. A key aspect of such studies is to obtain efficient and stable expression of a foreign antigen by a genetically engineered pathogen. The expression systems in Salmonella using full-length ovalbumin constructs available thus far have resulted in suboptimal responses in vivo [16-18]. The current investigation highlights the use of a novel construct based on the polymeric surface display of an ovalbumin reporter epitope to amplify the signal for the improved activation and detection of cognate CD4+ T cells.

\section{Results}

Construction, expression and export of the MisL-OVA-CD4 fusion proteins

The Salmonella autotransporter protein MisL was used to display a specific MHC class II ovalbumin peptide (OVA-CD4) on the surface of a Salmonella vaccine vector. The OVA-CD4 peptide was fused through genetic engineering to the $\mathrm{N}$-terminus of the translocator domain of MisL. The construct was based on the pnirLTBMisL plasmid [7] that encodes a truncated MisL protein with the signal sequence of the heat-labile enterotoxin of E. coli in frame. This truncated MisL lacks the aminoterminal 423 amino acids of the mature MisL. The expressed MisL protein included an additional 210 amino acids of its alpha domain to avoid potential protein misfolding in the periplasm and ensure correct conformation in the outer membrane for transit of the passenger domain to the bacterial surface and display of its $\mathrm{N}$-terminus $[7,8]$. The plasmid used the nirB promoter that is activated under the anaerobic conditions of the intestinal environment and induces improved systemic immune responses towards foreign antigen expressed by orally administered Salmonella vaccine strains [19-21]. This vector is not a high copy number plasmid as antibody responses against antigens delivered by Salmonella are improved when expressed from low to medium copy number plasmids that minimize metabolic stress upon the carrier strain [22,23]. Previous addition of the spiC promoter, which is activated in antigen-presenting cells, was used successfully to express the MisL protein or the 987P fimbriae of E. coli as viral epitope carriers on the surface of a Salmonella vaccine vector. Oral immunization experiments in mice also suggested improved in vivo expression by showing better humoral immune responses against the foreign antigens $[10,24]$. Thus, we prepared a similar promoter tandem construct by inserting the spiC promoter between the nirB promoter and the Shine Delgarno site for MisL transcription (Figure 1). Expression of the OVA-CD4MisL fusion protein was evaluated with Salmonella strain SL7207 carrying either pZS1202 (nirBp) or pZS1204 (nirBp and spiCp). Using an antibody specific for the OVA-CD4 epitope, the fusion protein of expected size (approximately $60 \mathrm{kDa}$ ) was detected by western blot analysis for both constructs with approximately three times more protein being expressed when both promoters were used, as determined by densitometry (Figure 2A). The OVA-CD4 specific antibody was confirmed to react with ovalbumin but not with Salmonella-expressing MisL alone. The detection of the fusion protein in Salmonella grown under aerobic conditions suggests some promoter leakiness, although the high affinity of the anti-OVA-CD4 antibody used in these assays might render detection very sensitive. Previous data indicated that magnesium activated fimbrial subunit gene expression from the spiC promoter in a phoP mutant [24]. However, using the same mutant strain, a significant effect due to magnesium was not detectable for MisL, possibly due to the fact that MisL is a much larger protein than the previously studied fimbrial subunit. In order to demonstrate that the OVA-CD4-MisL fusion protein was crossing the cytoplasmic membrane and behaved like the native MisL protein, outer membranes prepared from strain SL7207 carrying the plasmid with the misL fusion gene were analyzed by western blotting. The OVA-CD4-MisL fusion protein was detected in the outer membrane preparation using the ovalbumin- 


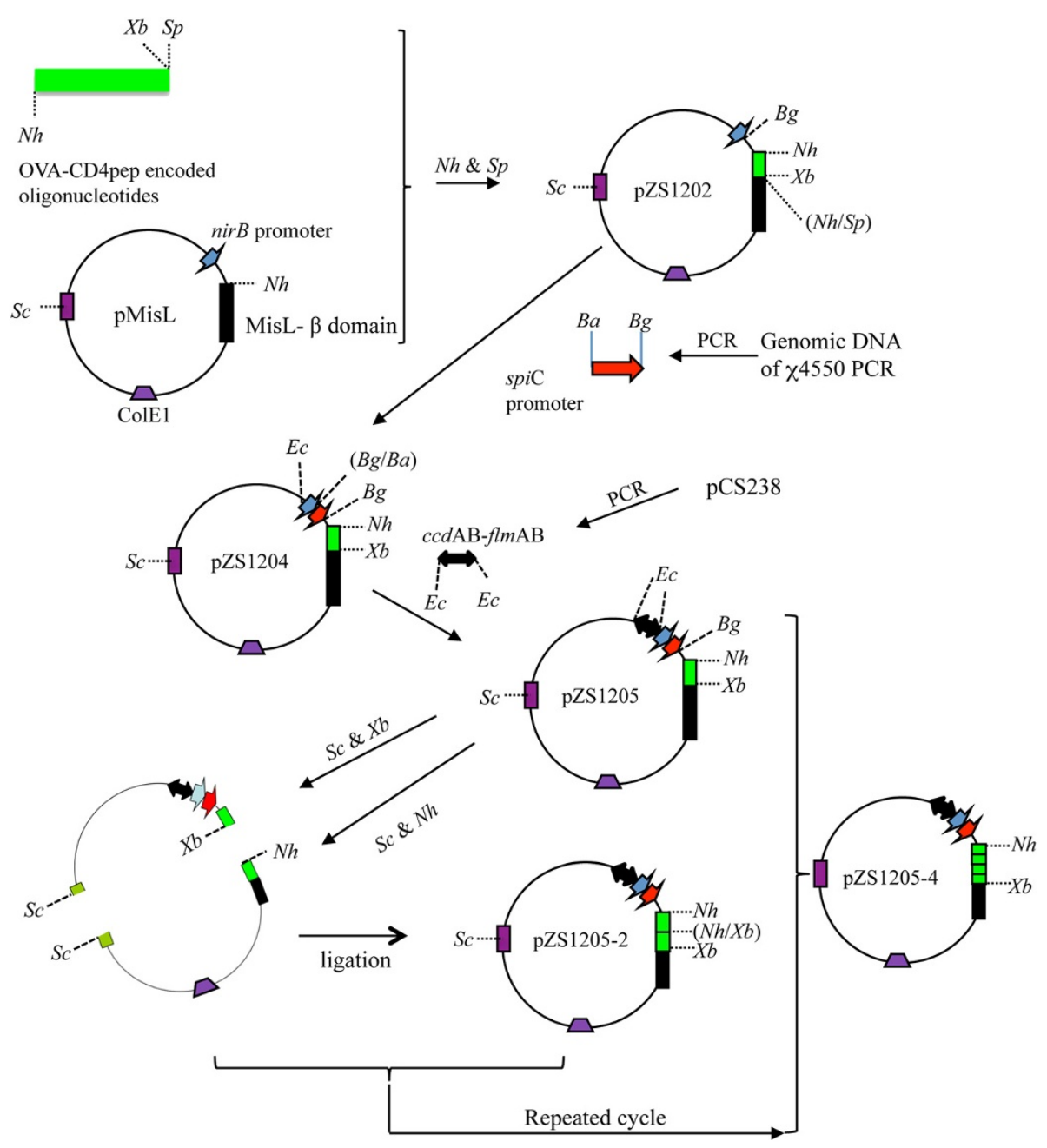

Figure 1 Plasmid constructions, as described in Methods.

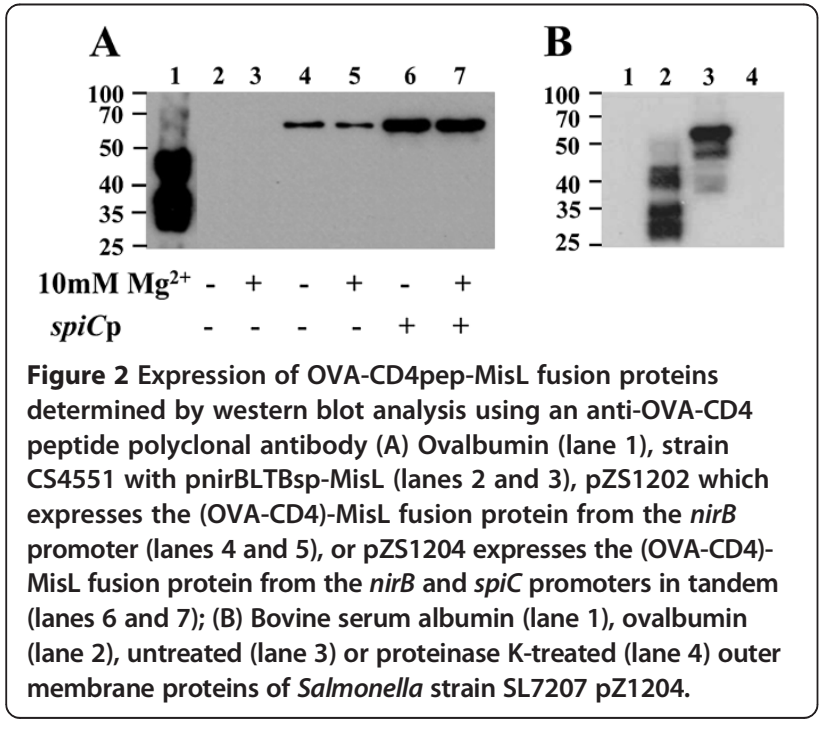

peptide specific antibody (Figure 2B), showing that the fusion protein was not affected in its export and localization properties. Moreover, fused OVA-CD4 peptide was susceptible to proteinase $\mathrm{K}$ degradation, characterizing its accessibility on either one or both outer membrane surfaces.

\section{Stabilized plasmids}

To ensure that attenuated Salmonella strains would keep the OVA-CD4-MisL plasmid construct in the absence of antibiotic selection after administration to mammalian hosts, two post-segregational killing systems from the $\mathrm{F}$ plasmid of $E$. coli, the $\operatorname{fim} A B$ and $c c d A B$ genes were engineered into the plasmid (Figure 1). Efficiency of plasmid maintenance was investigated by growing Salmonella SL7207 with or without the stabilized plasmid in antibiotic-free media for approximately 530 generations. The plasmid-bearing rate was calculated by dividing the number of bacteria recovered from 
antibiotic plates by the number of bacteria recovered from antibiotic-free plates. As shown in Figure 3, plasmid pZS1204 with no stabilization system was gradually lost during after 20 daily passages, however plasmid pZS1205 was kept in essentially all the bacteria. A derivative of the latter plasmid, pZS1205-4, which expresses four copies of the OVA-CD4 was 100\% maintained for one week and in nearly half of the bacteria for 26 days. In contrast, the high copy number plasmid pUC18-OVA that constitutively expressed the full-length ovalbumin protein was lost in five days. These results confirmed that high copy number plasmid constructs have the undesirable effect of being unstable in the absence of antibiotic selection. More importantly, the data demonstrated that the $\operatorname{fim} A B$ and $c c d A B$ genes have an efficient plasmid stabilization property that prolongs the life span of foreign antigenexpressing Salmonella.

\section{Surface exposure of the OVA-CD4 epitope peptide on Salmonella}

To determine whether the OVA-CD4 portion of the fusion protein was located on the periplasmic or extracellular face of the outer membrane, intact Salmonella expressing the fusion protein were subjected to treatments with various concentrations of proteinase K. Presence of the OVA-CD4 peptide was then determined by western blot analysis with the OVA-CD4 specific antibodies (Figure 4A). Parallel

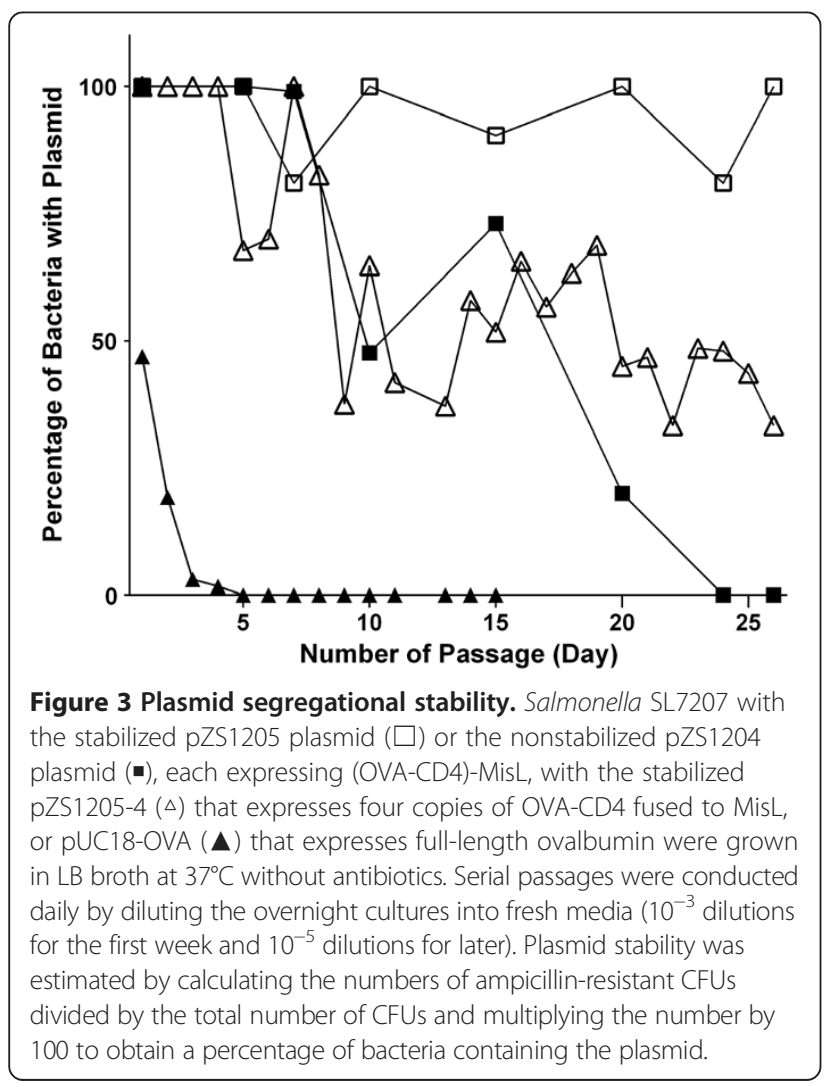

western blots were probed for the periplasmic $\beta$-lactamase with an anti-ampicillinase antibody to evaluate concentrations of proteinase $\mathrm{K}$ that would be effective on the bacterial surface without permeating into the periplasmic compartment (Figure 4B). Proteinase $\mathrm{K}$ at a concentration of $11 \mu \mathrm{g} / \mathrm{ml}$ completely degraded the OVA-CD4 peptide without affecting the periplasmic $\beta$-lactamase, indicating that the latter peptide was exposed on the bacterial surface. A comparable result was obtained with higher concentrations of proteinase $\mathrm{K}$, albeit a partial proteolytic effect on the $\beta$-lactamase suggested some permeation of the protease.

\section{Construction and display of oligomeric OVA-CD4 epitopes on the surface of Salmonella}

Having engineered OVA-CD4 encoded DNA to be flanked by restriction sites with matching $5^{\prime}$ extensions (XbaI and NheI) on plasmid pZS1205, it was possible to construct new homocomposite plasmids that add OVACD4 peptides in tandem to the amino-terminal end of MisL. We prepared plasmids capable of expressing MisL fusion proteins carrying dimeric and tetrameric OVACD4 epitopes and compared their levels of expression and surface display. Western blot analysis of these constructs showed that each addition of OVA-CD4 epitope copies to MisL significantly increased expression of the fusion protein (Figure 4C). Densitometry of the steadystate detectable proteins indicated that the (OVA-CD4) ${ }_{2}$-MisL dimer and (OVA-CD4) $)_{4}$-MisL tetramer constructs had approximately 2.7 and 4.8 times more OVACD4 epitopes, respectively, than the (OVA-CD4) ${ }_{1}$-MisL monomeric epitope fusion protein. We speculated that the lower molecular bands seen with the OVA tetramer were due to degradation of surface epitopes by the outer membrane protease PgtE [24], therefore we repeated the experiment with an insertion mutant of Salmonella SL7207 deficient for PgtE expression. Densitometry revealed greatly reduced degradation products for the tetramer construct and 1.7 and 4.4 times more OVACD4 epitopes were expressed with the dimer and tetramer MisL fusion proteins compared to the monomer construct (Figure 4D). All the ovalbumin epitopes expressed by Salmonella were accessible to the proteinase $\mathrm{K}$ proteolytic activity, consistent with their surfaceexposure. The increased numbers of smaller products for the tetramer construct in the absence of proteinase $\mathrm{K}$ pointed towards bacterial auto-degradation processes. Surface display of the OVA-CD4 epitope was visually confirmed by fluorescence microscopy. Using the OVACD4 specific antibody with a FITC-labeled secondary antibody to label the surface of Salmonella SL7207 expressing one, two or four copies of OVA-CD4 fused to MisL, the intensity of the fluorescence increased accordingly, as determined by the number of additional epitope 

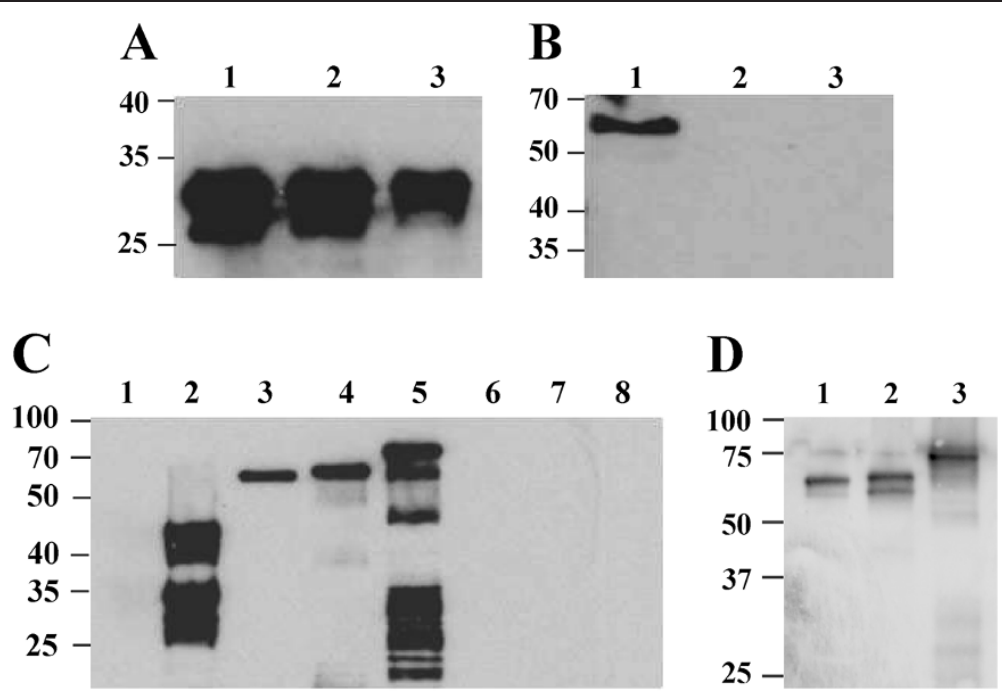

Figure 4 Surface exposure of (OVA-CD4)-MisL fusion protein detected by western blot analysis. Salmonella SL7207 pZS1205 treated with 0

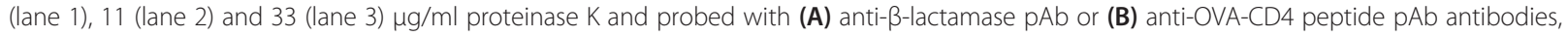
respectively. (C) Salmonella SL7207 expressing MisL fusion proteins with one (pZS1205, lanes 1 and 6), two (pZS1205-2, lanes 4 and 7) or four (pZS1205-4, lanes 5 and 8) copies of the OVA-CD4 epitope; untreated (lanes 3 to 5) or treated (lanes 6 to 8) with proteinase K. Bovine albumin (lane 1) and ovalbumin (lane 2), as negative and positive antibody controls. (D) Salmonella SL7207 pgtE expressing MisL fusion proteins with one (pZS1205, lane 1), two (pZS1205-2, lane 2) or four (pZS1205-4, lane 3) copies of the OVA-CD4 epitope.

copies (Figure 5). Fluorescence intensities per bacterial cells using the NIH ImageJ software suggested that the OVA-CD4 dimer and tetramer fusion constructs displayed respectively 2.1 and 3.4 times more epitope as compared to the monomer construct. Fluorescence intensities with the PgtE mutant containing the same plasmids showed similar increases in epitope expression, with 2.4 and 5 times more epitope displayed by the dimer and tetramer constructs respectively (data not shown). The incremental levels of fluorescence and western blot bands confirmed higher expression and surface exposure of the OVA-CD4 peptide with increased numbers of tandem repeats in the constructs.

\section{Expression of full-length ovalbumin versus (OVA-CD4) 4 -MisL}

The relative efficiency of OVA-CD4 epitope expression harboring pZS1205-4, which encodes the peptide tetramer fused to MisL, or pUC18-OVA, which encodes the fulllength protein [25], was compared by western blot analysis with the anti-OVA-CD4 antibody (Figure 6). E. coli was used as host to compare ovalbumin expression under
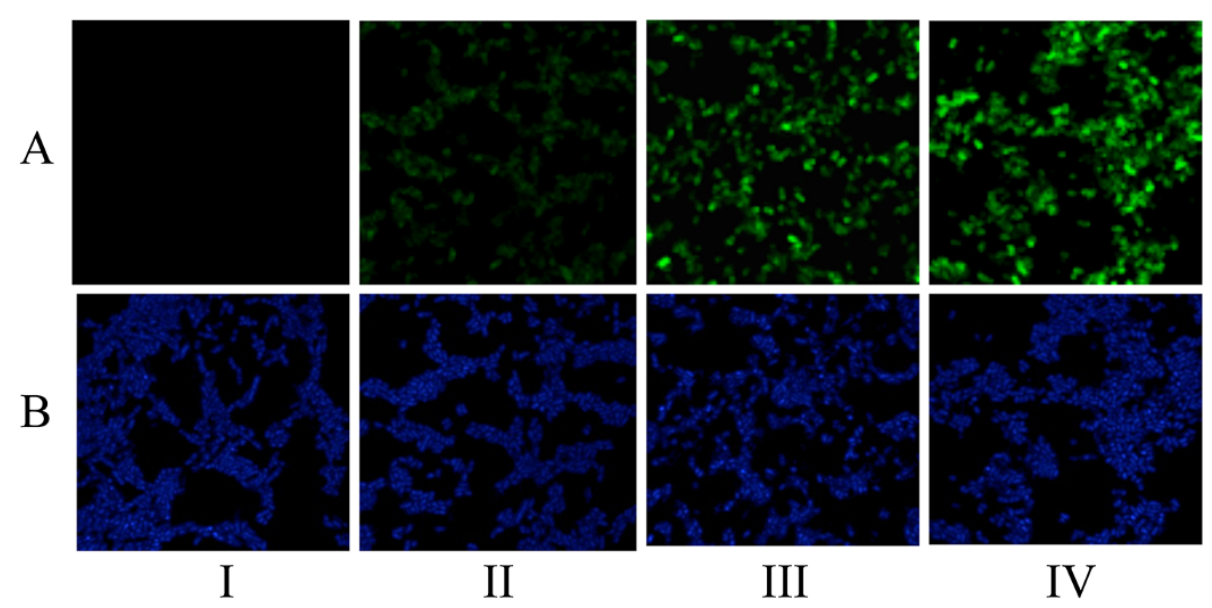

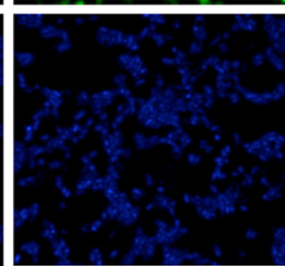

III

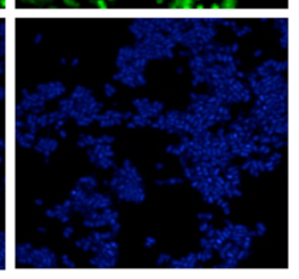

IV

Figure 5 Confocal microscopy of (A) Salmonella SL7207 labeled with rabbit anti-OVA-CD4 antibodies and FITC-conjugated anti-rabbit antibodies, and (B) DAPI-stained Salmonella SL7207. Salmonella SL7207 carried pnirBLTBsp-MisL (I), pZS1205 (II), pZS1205-2 (III) or pZS1205-4 (IV). 


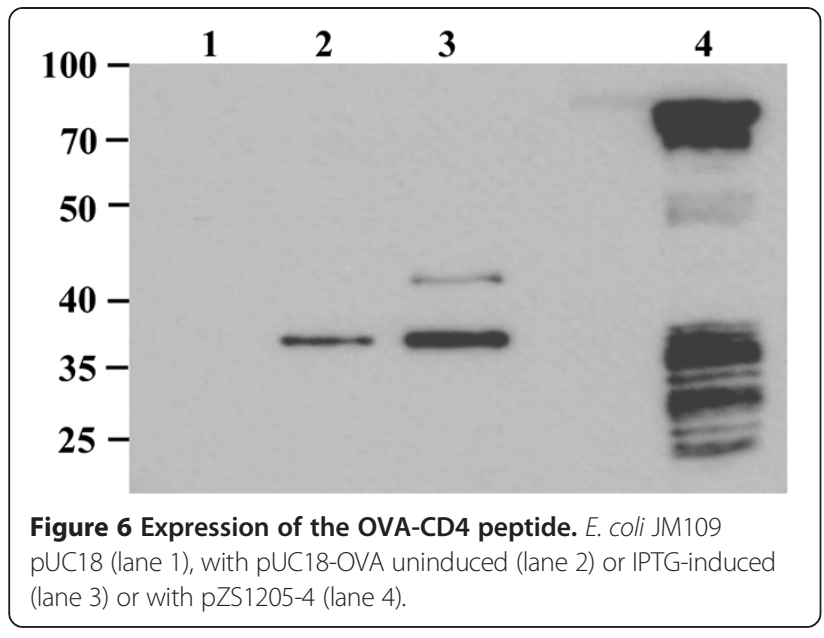

uninduced and induced conditions. The signal of the MisL fusion construct was 2.6 or 5.6 times stronger than the one from the full-length protein with or without IPTGinduction, respectively.

\section{In vitro antigen presentation}

The efficacy of the Salmonella SL7207pZS1205-4 [(OVACD4) ${ }_{4}$-MisL] constructs in stimulating ovalbumin specific $\mathrm{CD} 4 \mathrm{~T}$ cell responses was tested using in vitro antigen presentation assays with OTII TCR transgenic system. The responses obtained with the current construct were compared to a known full-length ovalbumin expressing system (pUC18-OVA) that has been used extensively in the field [25,26], using the same Salmonella strain. The OTII T cells were labeled with CFSE in these in vitro assays and the dilution of CFSE was used to measure $\mathrm{T}$ cell proliferation. When T cells were co-cultured with APCs pulsed with parental strains of Salmonella that do not express ovalbumin epitopes, they did not undergo proliferation as evident from the lack of CFSE dilution (Figure 7A). APCs pulsed with Salmonella expressing the full length ovalbumin or (OVA-CD4) $)_{4}$-MisL resulted in activation of T cells and their proliferation. The OTII T cell expansion was significantly higher in response to (OVA-CD4) $4_{4}^{-}$ MisL as compared to full-length ovalbumin, when each was expressed by the same Salmonella strain (Figure 7A and B).

\section{In vivo antigen presentation}

To test the efficacy of the various bacterial constructs in vivo, an adoptive transfer model was used, as described in the Methods section. The OTII T cells expressed the congenic marker CD45.1 and could thus be tracked in the recipient mice (CD45.2). Seven days after oral infection with the different bacterial strains, the mice were euthanized and lymphocytes were isolated from the spleen and mesenteric lymph nodes and analyzed by flow cytometry. These studies showed that there

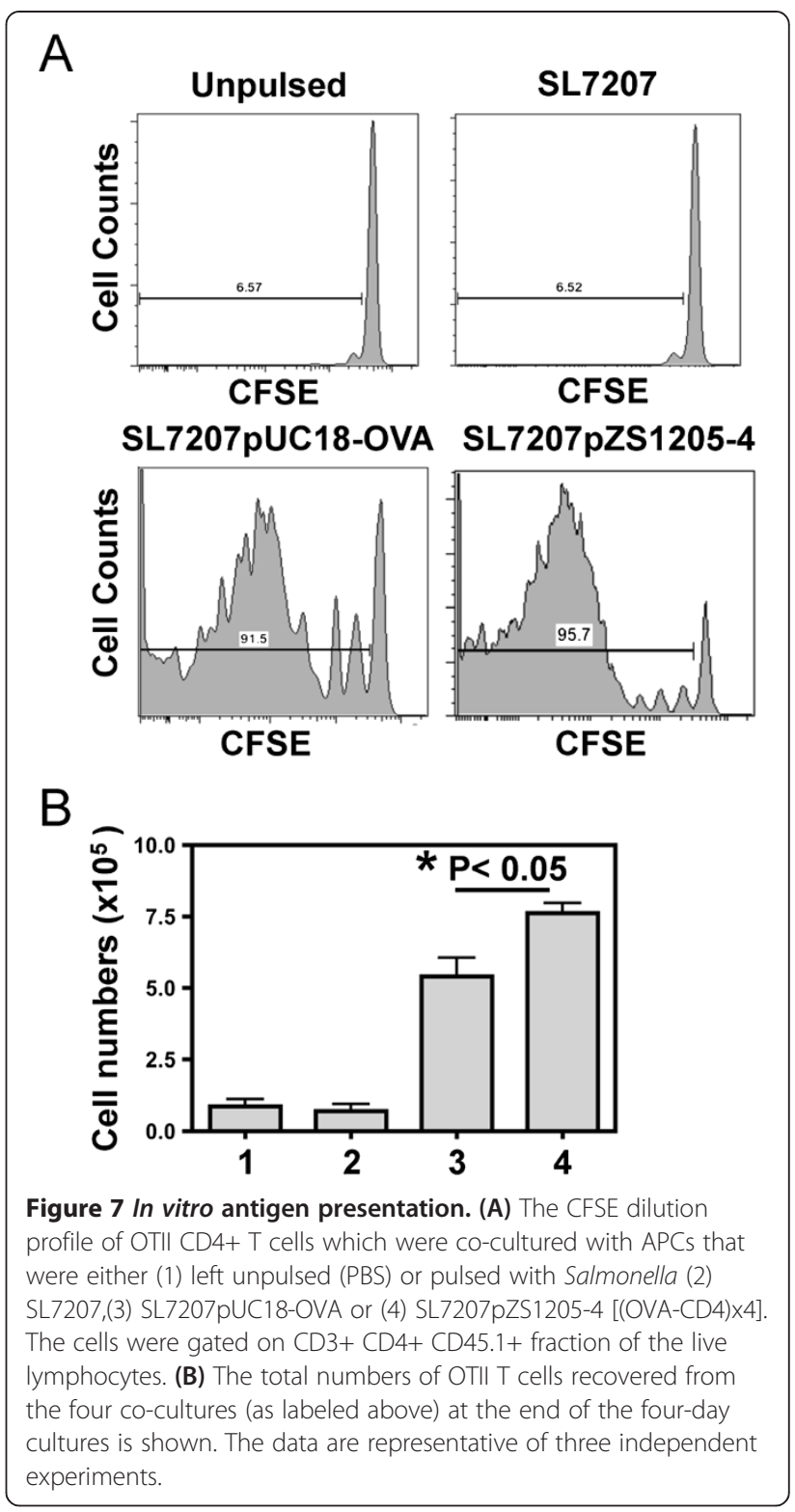

was an increase in the frequency of the OTII T cells during infection with the ovalbumin-expressing Salmonella SL7207 compared to the controls that received PBS or Salmonella alone (Figure 8A). The frequency of OTII T cells (CD45.1 CD4+) in the mice that were administered the strain expressing (OVA-CD4) $)_{4}$ was higher as compared to the mice infected with the strain that expressed full-length ovalbumin. No deletion of OVA-CD4 repeats was detected during the time of the in vivo experiment (data not shown). Based on the analysis of surface marker expression for activation, such as up regulation of CD44 and CD25, and down regulation of CD62L, there was a higher frequency of activated OTII T cells in the mice infected with the (OVA-CD4) $)_{4}$-expressing Salmonella (Figure 8B). These differences were also 


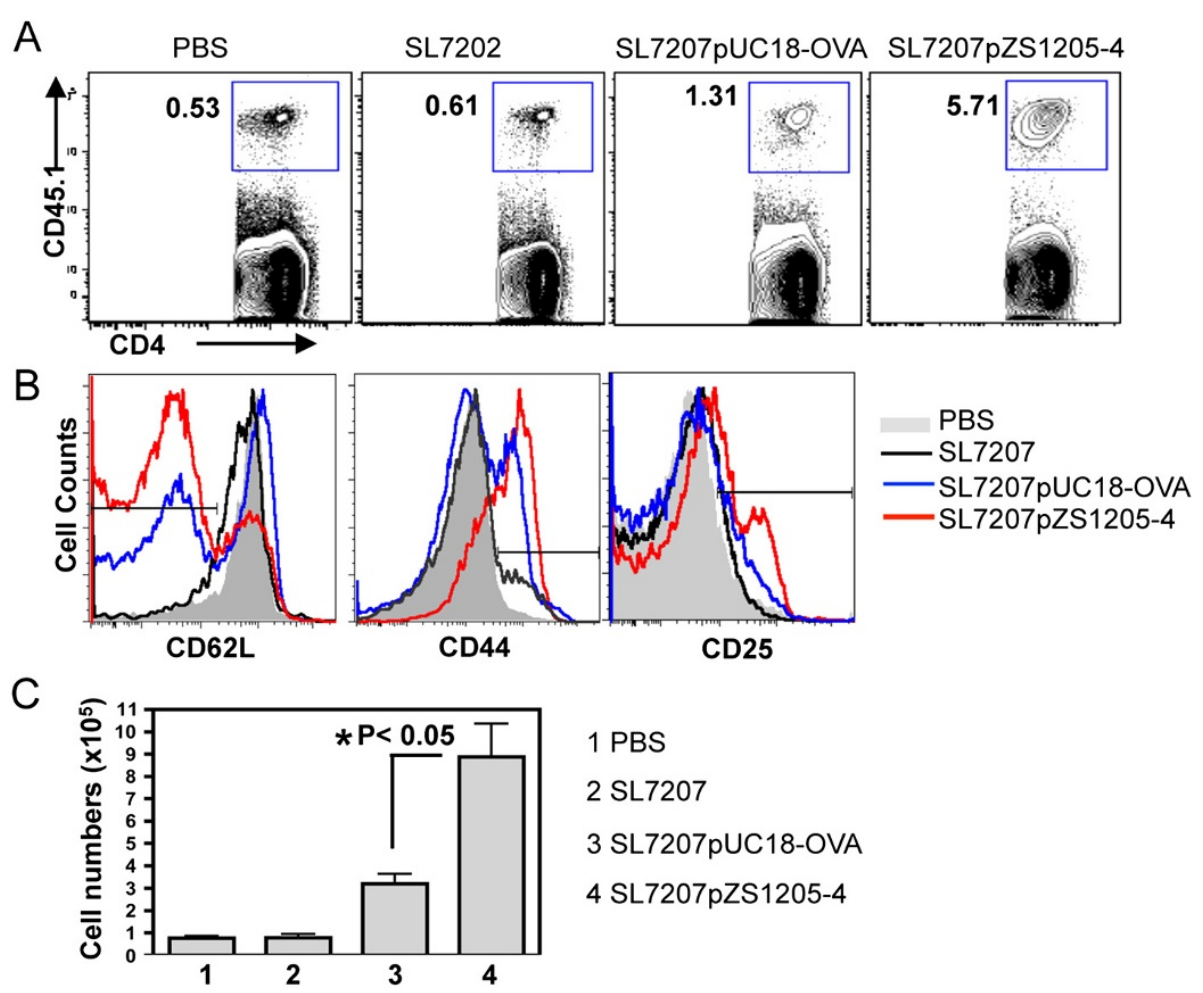

Figure $\mathbf{8}$ In vivo antigen presentation. (A) The frequency of OTII CD4+ T cells isolated from the mesenteric lymph nodes of mice that were administered orally (1) PBS, (2) Salmonella SL7207, (3) SL7207pUC18-OVA or (4) SL7207pZS1205-4 [(OVA-CD4)x4]. The cells were gated on CD4+ CD3+ live lymphocytes and the transferred T cells were identified using the congenic marker CD45.1. (B) The expression of the surface markers CD62L, CD44 and CD25 on the gated OTII T cells isolated from the four groups described above; PBS (grey area), Salmonella SL7207 (black line), SL7207pUC18-OVA (blue line) and SL7207pZS1205-4 [(OVA-CD4)x4] (red line). (C) The total numbers of OTII T cells recovered from the mesenteric lymph nodes of the four groups of mice described above. The data shown are representative of three independent experiments.

apparent in the total numbers of OTII T cells in the mesenteric lymph nodes (Figure $8 \mathrm{C}$ ) and spleens (data not shown), with significantly higher numbers of OTII T cells present in the mice infected with the (OVA-CD4) $)_{4}$-expressing Salmonella compared to the mice infected with the full-length ovalbumin-expressing Salmonella. Together these data showed that the newly developed construct permits robust expression of the model ovalbumin epitope both in vitro and in vivo and can be used as reliable model to track antigen specific $\mathrm{T}$ cell responses.

\section{Discussion}

Our studies demonstrated that the MisL autotransporter protein was capable of displaying the OVA-CD4 epitope on the surface of Salmonella when the epitope was grafted onto the amino-terminal side of MisL. Moreover, MisL fusion proteins engineered to carry the epitope in tandem copies increased the efficiency of surface display. Based on our previous work on foreign antigen expression by Salmonella, we designed a prototype plasmid to optimize in vivo antigen presentation. For this, a tandem in vivo inducible promoters was inserted upstream of the
OVA-CD4-MisL open reading frame in a low to medium copy number plasmid and two sets of stabilization systems were introduced into the construct. When compared to a traditional Salmonella construct expressing full-length ovalbumin in the cytoplasm from a multicopy number plasmid with a strong constitutive promoter, our prototype construct expressing four OVA-CD4 epitopes was significantly more efficient at presentation for activating CD4 T cells, both in vitro and in vivo.

A variety of microbes $[14,27,28]$, including wild type or attenuated entero-invasive Salmonella [16,25,29], have been genetically modified to express ovalbumin with its well-characterized CD4 and CD8 T cell epitopes as a reporter system to study cellular immune responses in hosts. Many of these studies did not attempt to optimize the expression level and bacterial delivery mechanism for the most efficient immune recognition of the relevant epitopes. For this study, we designed and tested an improved ovalbumin reporter construct based on previous studies with Salmonella vaccine vectors that have established general principles for the improvement of immune responses. First, surface display of a foreign epitope was reported to induce a better immune response 
than when the same epitope remained in the bacterial cytoplasm [20]. A recent study suggested that location on the bacterial surface was even more important for immunity than high abundance or immunodominance since Salmonella mainly survive in antigen-presenting cells, rendering surface antigens more accessible to the cell for processing and presentation [30]. Second, surface-exposed foreign epitopes presented as multimers induced better antibody responses than the same epitopes presented as monomers on a Salmonella vaccine vector [10]. Because it is capable of expressing antigens on a Salmonella surface, the MisL protein has the advantage of tolerating large fragments of proteins in a fusion construct. This property was used to incrementally add OVA-CD4 epitopes to MisL and demonstrated a corresponding stepwise increase of epitope expression on the surface of Salmonella. The addition of tandem epitope copies did not affect bacterial growth. Although surface expression of larger amounts of foreign epitopes can induce some degradation by the PgtE protease of Salmonella, as shown previously [24] and here with four copies of the OVA-CD4 epitope, PgtE did not significantly affect steady-state expression of the surface exposed epitope. A third generic rule to improve antigen delivery for better immune responses is the avoidance of high copy number plasmids that express large foreign antigen from constitutive promoters [23,31]. Such vector constructs are metabolic stressors for the bacteria and are rapidly eliminated or mutated, resulting in reduced levels of immune response [22]. In general, metabolic stress can be minimized by both the use of a low copy number plasmid and the expression of the smallest protein fragment needed for an experimental task, such as a major immunogenic epitope fused to a Salmonella protein. Here, we demonstrated that the multicopy number plasmid pUC18-OVA that expresses the full-length ovalbumin was lost in vitro after only a few passages in the absence of antibiotic selection, suggesting that it is not an optimal construct. In contrast, the low copy number pZS1204 was far more stable than pUC18-OVA. Safe remedies to stability issues for the expression of foreign proteins have been the use of chromosomal integration or single-copy number plasmids together with strong constitutive or in vivo inducible promoters [32-34]. As an alternative to stabilize protein expression, one can borrow plasmid post-segregational killing genes. We used the latter approach by inserting a cassette comprising two of these set of genes that we have previously used with success [10] to obtain pZS1205 and confirmed significantly improved plasmid stability over 500 generations. In addition, approaches shown to augment the expression of ovalbumin or other foreign antigens in animal models were based on the engineering of in vivo-activated promoters [35-37]. For this study, we took advantage of a tandem promoter system that we had previously shown to express antigen efficiently both in vitro and in vivo [10,24].
The improved efficiency of antigen presentation with the engineered Salmonella displaying stably several copies of the OVA-CD4 epitope on its surface was demonstrated here by the enhanced CD4+ T cell multiplication both in vitro and in vivo, using a classical bacterial fulllength ovalbumin construct in the same bacterial background for comparison. Although we did not evaluate the individual effect of each construction step on this immune response, we used a systematic approach based on an extensive body of literature and our previous studies to engineer an efficient ovalbumin reporter system. Among the many autotransporter proteins that are capable of presenting heterologous surface antigens or secreted proteins, MisL has already demonstrated its adaptability as an antigen delivery system in the context of experimental Salmonella vaccines [7-10,38]. Some of these studies showed that MisL was tolerant to short epitope multimers. Here, we confirmed multimer display with a longer epitope fused to MisL and demonstrated efficient activation of CD4+ T cells. The identification of an increasingly larger panoply of autotransporter proteins capable of exporting foreign proteins might help to partially bypass display or secretion limitations due to the nature, size and number of epitopes added $[4,39]$. Although the construct described here was developed to create an improved ovalbumin reporter system for the study of CD4+ T cell responses to Salmonella, the concept of epitope presentation in the form of tandemly repeated homopolymers might be applicable for other purposes, including their cytoplasmic delivery by a bacterial injection apparatus or invasive mechanism for a CD8+ T response $[10,40,41]$. Polymerization of protective epitopes should also benefit vaccine development.

\section{Methods}

\section{Bacterial strains, media and reagents}

The bacterial strains used in this study were as follows: E. coli DH5 alpha $\left(\mathrm{F}^{-}\right.$\%80lacZ $\Delta \mathrm{M} 15 \Delta$ (lacZYA-argF) $\mathrm{U} 169$ recA1 endA1 hsdR17( $\left.\mathrm{rk}^{-}, \mathrm{mk}^{+}\right)$phoA supE44 thi-1 gyrA96 relA1 $\lambda^{-}$) [42], JM109 [43], Salmonella enterica serovar Typhimurium SL7207 (hisG46, DEL407 [aroA544::Tn10 $\left.\left(\mathrm{Tc}^{\mathrm{s}}\right)\right]$ )(derivative of strain SL3261, kind gift from Dr. Bruce Stocker, Stanford University) [44], $S$. Typhimurium $\mathrm{X} 4550$ (gyrA1816 $\operatorname{asdA1} \Delta($ zhf-4::Tn10) $\Delta$ crp-1 $\Delta$ cya-1) [45], S. Typhimurium CS4551 ( 45550 phoP::cat) and S. Typhimurium CS4552 (X4550 pgtE:: aphA) [24]. The SL7207 pgtE mutant was engineered by generalized transduction, using phage P22. Successful transfer was confirmed by PCR. All the strains were grown in Luria-Bertani (LB, Lennox) medium (Difco, Detroit, MI, USA), with the addition of $50 \mu \mathrm{g} / \mathrm{ml} \mathrm{DL-}$ alpha, E-diaminopimelic acid (DAP; Sigma, St. Louis, Mo.) for S. Typhimurium strains $\chi^{4550}$ and CS4551. Media were supplemented with ampicillin $(100 \mu \mathrm{g} / \mathrm{ml})$, 
Table 1 List of plasmids

\begin{tabular}{|c|c|c|c|}
\hline Plasmids & Characteristics & Resistance & References \\
\hline$\overline{p G E M^{\oplus}-T}$ & & $A p^{r}$ & Promega, Madison, Wl \\
\hline pUC18-OVA & $1.2 \mathrm{~kb}$ ovalbumin gene in pUC18 & $A p^{r}$ & {$[25]$} \\
\hline pZS1201 & pGEM ${ }^{\circledast}-T$ Easy Vector - OVA-CD4 & $A p^{r}$ & This study \\
\hline pnirBLTBsp-MisL & pnirB for LTsp-MisL, colE7-like ori & $A p^{r}$ & [7] \\
\hline pZS1202 & pnirBLTBsp-MisL-OVA-CD4 & $A p^{r}$ & This study \\
\hline pZS1203 & pGEM ${ }^{\circledR}-T$ Easy Vector -spiCp & $A p^{r}$ & This study \\
\hline pZS1204 & pZS1202 with spiCp & $A p^{r}$ & This study \\
\hline pCS238 & pACYC184 with $c c d A B-f / m A B$ & $\mathrm{Cm}^{r}$ & [10] \\
\hline pZS1205 & pZS1204 with $c C d A B-f I m A B$ & $A p^{r}$ & This study \\
\hline pZS1205-2 & pZS1205 with [OVA-CD4]×2 & $A p^{r}$ & This study \\
\hline pZS1205-4 & pZS1205 with [OVA-CD4]x4 & $A p^{r}$ & This study \\
\hline
\end{tabular}

chloramphenicol $(30 \mu \mathrm{g} / \mathrm{ml})$, kanamycin $(50 \mu \mathrm{g} / \mathrm{ml}), \mathrm{X}-$ Gal $(35 \mu \mathrm{g} / \mathrm{ml})$, and IPTG $(0.5 \mathrm{mM})$, or $\mathrm{MgCl}_{2}$ overnight [24] when needed or indicated. Restriction and modification enzymes were from New England Biolabs Inc. (Beverly, MA, USA). Unless specified otherwise, reagents were purchased from Sigma.

\section{Plasmid constructs}

Table 1 lists all the plasmids used in this study. Plasmids carrying the genes for MisL-[OVA-CD4 $]_{\mathrm{n}}(\mathrm{n}=1,2$ or 4$)$ fusion proteins were engineered to be under the control of two in vivo-inducible promoters in tandem (Figure 1) [24]. For this, both strands for the ovalbumin derived CD4 epitope (OVA-CD4: "ISQAVHAAHAEI NEAGR") were synthesized as follows: Forward strand: 5' -GCTAGCGGTGGCATTAGCCAGGCCGTGCATGC GGCCCATGCGGAAATTAACGAAGCCGGCCGCGGT GGCTCTAGAACTAGTA-3'; Reverse strand: 5' -ACT AGTTCTAGAGCCACCGCGGCCGGCTTCGTTAAT TTCCGCATGGGCCGCATGCACGGCCTGGCTAATG CCACCGCTAGCA-3'. Both strands had an NheI restriction site at one end, and $\mathrm{XbaI}$ and SpeI restriction sites at the other end. The $5^{\prime}$ ends were phosphorylated and the 3' end had an additional A for TA cloning. The hybridized strands were ligated into the $\mathrm{pGEM}^{\circ} \mathrm{T}$ easy vector (Promega), resulting in plasmid pZS1201. The OVA-CD4 epitope containing fragment from NheI and SpeI, restricted pZS1201 was purified by agarose gel electrophoresis and ligated into the NheI restriction site of pnirBLTBsp-MisL [7], resulting in plasmid pZS1202. The spiC promoter from strain $\mathrm{X} 4550$ was amplified by $\mathrm{PCR}$, using upper primer $5^{\prime}$ - ATGCGGATCCAATGCTTCCC TCCAGTTGCCTGTT-3' and lower primer 5'-ATGCGGAGATCTAAATGGGAGTTTCTATCAAATTC-3', carrying a BamHI or BglII near their $5^{\prime}$ end, [24]. The amplicon ligated into $\mathrm{pGEM}^{\circ} \mathrm{T}$ was designated pZS1203. The BamHI BglII fragment of pZS1203 carrying the spiC promoter was ligated into the $B g l I I$ site of pZS1202, which is downstream of the nirB promoter sequence [21], resulting in pZS1204, as plasmid with tandem promoters. Two post-segregational killing systems $(c c d \mathrm{AB}$ and $f l m \mathrm{AB})$ were amplified by PCR using pCS238 as template and primers 5'-ATCGTGAATTCCTGCAGACTGGCTGTG TATAAC-3' and 5' -ATCGTGAATTCCCTGGCAGTCT GGTTGTTCAT-3'[10]. The amplicon was restricted with EcoRI and inserted into the corresponding site of pZS1204, creating plasmid pZS1205. A second OVA-CD4 epitope was added to the latter plasmid by restricting pZS1205 with NheI and ScaI or with XbaI and ScaI, and the two fragments containing the DNA encoding for the OVA-CD4 epitope were purified by agarose gel electrophoresis and ligated to create pZS1205-2. This procedure was repeated to obtain pZS1205-4, which carries 4 tandem copies of OVA-CD4 DNA as an in-frame fusion to MisL. All the amplified and cloned PCR products were checked for sequence accuracy.

\section{Preparation of outer membrane proteins}

Outer membrane fractions were prepared from spheroplasts, as described previously [46]. Bacteria from $10 \mathrm{ml}$ overnight cultures (approximately $10^{10} \mathrm{CFU}$ ) were pelleted by centrifugation and suspended in $90 \mu \mathrm{l}$ of $30 \mathrm{mM}$ Tris- $\mathrm{HCl}$ (pH 8.0) with $20 \%$ sucrose. $10 \mu \mathrm{l}$ of lysozyme ( $1 \mathrm{mg} / \mathrm{ml}$ in $0.1 \mathrm{M}$ EDTA) was added and the bacteria were incubated for $30 \mathrm{~min}$ on ice. The obtained spheroplasts were stabilized with $\mathrm{MgCl}_{2}(20 \mu \mathrm{M}$ final concentration) and centrifuged $(16,000 \times \mathrm{g}, 2 \mathrm{~min})$. The periplasmic proteins were removed with the supernatant and the spheroplasts were resuspended in $100 \mu \mathrm{l}$ of a $10 \mathrm{mM}$ Tris- $\mathrm{HCl}$ (pH8.0), $100 \mathrm{mM} \mathrm{NaCl}, 10 \mathrm{mM}$ $\mathrm{MgCl}_{2}$ solution containing $1 \mu \mathrm{g}$ of DNase per ml, and lysed by sonication (two times $1 \mathrm{~min}$ with a Cup Horn accessory at amplitude output 10, using a model XL2020 sonicator; Heat System, Farmingdale, N.Y.). Cytoplasmic membrane proteins were solubilized by incubating the membranes for $20 \mathrm{~min}$ at room temperature with $\mathrm{N}$ - 
lauroylsarcosine, sodium salt (ICN Biochemicals, Cleveland, Ohio) at a final concentration of $0.5 \%$ [47]. Residual intact cells were removed by centrifugation at $1,200 \times \mathrm{g}$ for $10 \mathrm{~min}$ at $4^{\circ} \mathrm{C}$. The non-solubilized outer membranes were pelleted by high-speed centrifugation in a Beckman JA-18.1 rotor at $17,000 \mathrm{rpm}$ for $3.0 \mathrm{~h}$ at $4^{\circ} \mathrm{C}$. The outer membrane pellet was suspended in $200 \mu \mathrm{l}$ PBS and mixed 1:1 with $2 \times$ sodium dodecyl sulfate-polyacrylamide gel electrophoresis (SDS-PAGE) buffer (sample buffer).

\section{Protease accessibility to surface exposed domains of MisL}

\section{fusion proteins}

Bacteria grown to log phase $\left(\mathrm{A}_{600} \sim 0.6\right)$ were washed in PBS three times, then divided in four samples. Proteinase K (recombinant, Roche Diagnostics GmbH, Mannheim, Germany) was added to final concentrations of 11,33 or $100 \mu \mathrm{g} / \mathrm{ml}$, leaving one sample as negative control. The samples were incubated at $37^{\circ} \mathrm{C}$ for $30 \mathrm{~min}$, and protease activity was stopped by adding AEBSF [4-(2-Aminoethyl) benzenesulfonyl fluoride hydrochloride] to each sample at final concentration of $0.5 \mathrm{mM}$. The samples were directly mixed with $2 \times$ sample buffer, heated for $10 \mathrm{~min}$ at $100^{\circ} \mathrm{C}$, and $5 \mu \mathrm{l}$ per lane were analyzed by SDS-PAGE and western blotting.

\section{SDS-PAGE and western blotting}

For SDS-PAGE and western blotting, bacteria grown to log-phase $\left(\mathrm{A}_{600} \sim 0.6\right)$ were pelleted (except for the experiments with isolated outer membranes or using proteinase $K$, as described above), solubilized in sample buffer and boiled for $5 \mathrm{~min}$. Proteins were separated by SDS-PAGE. The gels were analyzed by western blotting, using affinity purified rabbit anti-OVA-CD4 epitope (ISQAVHAAHAEINEAGR) polyclonal antibodies (OVA 323-339, Innovagen, Lund, Sweden) diluted 1:1000 in PBS-0.1\% tween 20 (PBS-T) or with rabbit antiampicillinase antibodies (5 prime to 3 prime, Inc. Boulder, $\mathrm{CO}$ ), followed by horseradish peroxidase (HRP)-conjugated secondary antibodies diluted 1:8000 in PBS-T and enhanced chemiluminescence (ECL) for detection, as described previously [46]. Relative amounts of expressed fusion proteins were evaluated by densitometry, using $\mathrm{NIH}$ ImageJ software version 1.47n (http://rsb.info.nih.gov/ij/).

\section{Indirect immunofluorescence assay (IFA)}

For IFA, the bacterial strains were spread on glass slides, fixed with cold methanol and incubated with rabbit antiovalbumin antibody. The slides were washed three times with PBS-T, incubated with FITC conjugated goat antirabbit-IgG and washed again with PBS-T. Dried slides were mounted with $10 \mu \mathrm{l}$ antifade reagent with DAPI, overlaid with glass coverslip, and images were acquired on a Leica TCS SP5 inverted confocal microscope with a $63 \times(1.2 \mathrm{NA})$ water immersion lens. Antibody staining was visualized with $488 \mathrm{~nm}$ excitation from an Argon laser and DAPI was excited with a $405 \mathrm{~nm}$ pulsed diode laser. Relative fluorescence intensities were calculated from measurements with the NIH ImageJ software.

\section{Plasmid stability}

To determine the persistence of a plasmid in dividing cells, bacteria were passaged in LB broth without antibiotics for 26 days (approximately 530 generations) using daily $10^{-3}$ dilutions of overnight cultures for the first week and $10^{-5}$ dilutions for the remainder. Plasmid stability was estimated by determining at several passage points the numbers of antibiotic-resistant live bacteria containing the plasmid (colony-forming units or CFUs on ampicillin-containing LB agar plates) divided by the total number of live bacteria (CFUs on LB agar plates). This number was multiplied by 100 to obtain a percentage of bacteria containing the plasmid [10].

\section{Mice}

OTII TCR transgenic mice on a CD45.1 background were maintained in a specific pathogen-free (SPF) facility in the Department of Pathobiology at the University of Pennsylvania in conformance with institutional guidelines for animal care. C57BL/6 mice were purchased from Taconic farms and housed within the SPF facility. All animal studies were carried out in compliance with the guidelines of the Institutional Animal Care and Use Committee of the University of Pennsylvania.

\section{OTII T cell isolation}

CD4+ T cells were enriched from lymphocytes isolated from the spleen and pooled peripheral lymph nodes of OTII TCR transgenic mice (Ovalbumin specific CD4+ TCR transgenic mice) using CD4+ MACs beads (Miltenyii Biotech). The cells were labeled with the cytoplasmic dye CFSE (carboxyfluorescein diacetate succinimidyl ester, Molecular Probes, Eugene, OR), to track proliferation and used for the in vitro assays or transferred into mice for testing in vivo efficacy of the bacterial constructs.

\section{In vitro antigen presentation assay}

Antigen-presenting cells were isolated from spleens of WT mice; CD11C + cells were enriched from RBC lysed splenocytes using CD11c + MACs beads (Miltenyii Biotech). The purified APCs were resuspended in complete RPMI (10\% FBS) and plated in 24 well plates $\left(1 \times 10^{5}\right.$ cells/well). The APCs were pulsed with the various bacterial constructs at a multiplicity of infection (MOI) of 10 for 2 hours, following which the cells were washed to remove the extracellular bacteria, treated with gentamicin and used for the co-culture studies. The ability of the pulsed APCs to process and present the ovalbumin derived from the bacteria on MHC II molecules for $\mathrm{T}$ 
cell activation, was determined by co-culturing them with CFSE labeled OTII T cells $\left(5 \times 10^{5}\right.$ cells/well $)$ for a period of 4 days, as described previously [14]. At the end of the culture period, the cells were counted and stained for flow cytometric analysis.

\section{In vivo antigen presentation assay}

$2 \times 10^{6}$ purified OT1I cells (CD45.1) were adoptively transferred (intravenously) into B6 (CD45.2) recipient mice and the mice were infected orally 24 hours later. For infections, log phase cultures of the various bacterial strains were washed and re-suspended in PBS and administered by oral gavage ( $10^{9}$ bacteria/mouse) $10 \mathrm{mi}$ nutes before infection mice were given $0.1 \mathrm{ml}$ of $5 \%$ $\mathrm{NaHCO}_{3}$ to neutralize the stomach acidity. The mice were sacrificed on day 7 post infection and the spleens and mesenteric lymph nodes were harvested for flow cytometric analysis.

\section{Flow-cytometry}

Lymphocytes were isolated from spleens and cervical lymph nodes by mechanical homogenization followed by lysis of RBCs (for spleens) using lysis buffer $(0.846 \%$ $\mathrm{NH}_{4} \mathrm{Cl}$ ). Freshly isolated cells were stained with the antibodies purchased from eBioscience (San Diego, CA) or BD Biosciences (San Jose, CA). The stained samples were run on a FACSCanto (BD, San Jose, CA) and results were analyzed using FlowJo software (TreeStar Inc., Ashland, OR).

\section{Statistical significance}

Statistical significance of differences between the various groups was tested using the student's $t$ test and $\mathrm{p}<0.05$ was considered significant.

\section{Competing interests}

The authors declare that they have no competing interests.

\section{Authors' contributions}

JZ carried out all the genetic engineering. JZ and LDM carried out the protein expression studies. BJ carried out the cellular immunoassays and animal studies. DMS, WC and BJ conceived the study. DMS and BJ designed the experiments, and drafted the manuscript. All authors contributed to the preparation of the manuscript. All authors read and approved the final manuscript.

\section{Acknowledgments}

This research was supported by NIH grant R21 Al090234 (to BJ), and by $\mathrm{NIH}$ grant R21 Al098041, USDA grant 2013-67015-21285, a University of Pennsylvania Research Foundation grant and Research Initiative Funds from the University of Pennsylvania Veterinary Center for Infectious Disease (to DMS).

\section{Author details}

${ }^{1}$ State Key Laboratory of Agrobiotechnology and College of Biological Sciences, China Agricultural University, Beijing 100193, People's Republic of China. ${ }^{2}$ Department of Pathobiology, University of Pennsylvania School of Veterinary Medicine, Philadelphia, Pennsylvania 19104, USA. ${ }^{3}$ College of Food and Biological Engineering, Zhengzhou University of Light Industry, Zhengzhou, Henan province 450002, People's Republic of China.
Received: 29 September 2013 Accepted: 19 May 2014

Published: 4 June 2014

\section{References}

1. Hagan $\mathrm{CL}$, Silhavy TJ, Kahne D: beta-Barrel membrane protein assembly by the Bam complex. Annu Rev Biochem 2011, 80:189-210.

2. Rossiter $A E$, Leyton $D L$, Tveen-Jensen $K$, Browning DF, Sevastsyanovich $Y$, Knowles TJ, Nichols KB, Cunningham AF, Overduin M, Schembri MA, Henderson IR: The essential beta-barrel assembly machinery complex components BamD and BamA are required for autotransporter biogenesis. J Bacteriol 2011, 193:4250-4253.

3. Grijpstra J, Arenas J, Rutten L, Tommassen J: Autotransporter secretion: varying on a theme. Res Microbiol 2013, 164:562-582.

4. Jong WS, Sauri A, Luirink J: Extracellular production of recombinant proteins using bacterial autotransporters. Curr Opin Biotechnol 2010, 21:646-652.

5. van Bloois E, Winter RT, Kolmar H, Fraaije MW: Decorating microbes: surface display of proteins on Escherichia coli. Trends Biotechnol 2011, 29:79-86.

6. Dorsey CW, Laarakker MC, Humphries AD, Weening EH, Baumler AJ: Salmonella enterica serotype Typhimurium MisL is an intestinal colonization factor that binds fibronectin. Mol Microbiol 2005, 57:196-211.

7. Ruiz-Perez F, Leon-Kempis R, Santiago-Machuca A, Ortega-Pierres G, Barry E, Levine M, Gonzalez-Bonilla C: Expression of the Plasmodium falciparum immunodominant epitope (NANP)(4) on the surface of Salmonella enterica using the autotransporter MisL. Infect Immun 2002, 70:3611-3620.

8. Ruiz-Olvera P, Ruiz-Perez F, Sepulveda NV, Santiago-Machuca A, Maldonado-Rodriguez R, Garcia-Elorriaga G, Gonzalez-Bonilla C: Display and release of the Plasmodium falciparum circumsporozoite protein using the autotransporter MisL of Salmonella enterica. Plasmid 2003, 50:12-27.

9. Alvarez AM, Vaquero-Vera A, Fonseca-Linan R, Ruiz-Perez F, Villegas-Sepulveda N, Ortega-Pierres G: A prime-boost vaccination of mice with attenuated Salmonella expressing a 30-mer peptide from the Trichinella spiralis gp43 antigen. Vet Parasitol 2013, 194:202-206.

10. Chen H, Schifferli DM: Comparison of a fimbrial versus an autotransporter display system for viral epitopes on an attenuated Salmonella vaccine vector. Vaccine 2007, 25:1626-1633.

11. Griffin AJ, McSorley SJ: Development of protective immunity to Salmonella, a mucosal pathogen with a systemic agenda. Mucosal Immunol 2011, 4:371-382.

12. Jenkins MK, Khoruts A, Ingulli E, Mueller DL, McSorley SJ, Reinhardt RL, Itano A, Pape KA: In vivo activation of antigen-specific CD4 T cells. Annu Rev Immunol 2001, 19:23-45.

13. John $B$, Weninger $W$, Hunter CA: Advances in imaging the innate and adaptive immune response to Toxoplasma gondii. Future Microbiol 2010, 5:1321-1328.

14. Pepper M, Dzierszinski F, Crawford A, Hunter CA, Roos D: Development of a system to study $\mathrm{CD} 4+-\mathrm{T}$-cell responses to transgenic ovalbumin-expressing Toxoplasma gondii during toxoplasmosis. Infect Immun 2004, 72:7240-7246.

15. Pape KA, Kearney ER, Khoruts A, Mondino A, Merica R, Chen ZM, Ingulli E, White J, Johnson JG, Jenkins MK: Use of adoptive transfer of T-cell-antigen-receptor-transgenic T cell for the study of T-cell activation in vivo. Immunol Rev 1997, 156:67-78.

16. Chen ZM, Jenkins MK: Clonal expansion of antigen-specific CD4 T cells following infection with Salmonella typhimurium is similar in susceptible (Itys) and resistant (Ityr) BALB/c mice. Infect Immun 1999, 67:2025-2029.

17. Ravindran R, McSorley SJ: Tracking the dynamics of T-cell activation in response to Salmonella infection. Immunology 2005, 114:450-458.

18. Bumann D: T cell receptor-transgenic mouse models for studying cellular immune responses to Salmonella in vivo. FEMS Immunol Med Microbiol 2003, 37:105-109.

19. Londono LP, Chatfield S, Tindle RW, Herd K, Gao XM, Frazer I, Dougan G: Immunisation of mice using Salmonella typhimurium expressing human papillomavirus type 16 E7 epitopes inserted into hepatitis B virus core antigen. Vaccine 1996, 14:545-552.

20. Chen H, Schifferli DM: Mucosal and systemic immune responses to chimeric fimbriae expressed by Salmonella enterica Serovar Typhimurium vaccine strains. Infect Immun 2000, 68:3129-3139. 
21. Oxer MD, Bentley CM, Doyle JG, Peakman TC, Charles IG, Makoff AJ: High level heterologous expression in E. coli using the anaerobically-activated nirB promoter. Nucleic Acids Res 1991, 19:2889-2892.

22. Galen JE, Curtiss R III: The delicate balance in genetically engineering live vaccines. Vaccine 2013, published on line December 23, 2013; http://dx.doi org/10.1016/j.vaccine.2013.12.026.

23. Galen JE, Wang JY, Chinchilla M, Vindurampulle C, Vogel JE, Levy $H$, Blackwelder WC, Pasetti MF, Levine MM: A new generation of stable, nonantibiotic, low-copy-number plasmids improves immune responses to foreign antigens in Salmonella enterica serovar Typhi live vectors. Infect Immun 2010, 78:337-347.

24. Chen H, Schifferli DM: Construction, characterization and immunogenicity of an attenuated Salmonella enterica Serovar Typhimurium pgtE vaccine expressing fimbriae with integrated viral epitopes from the spiC promoter. Infect Immun 2003, 71:4664-4673.

25. Wick MJ, Pfeifer JD: Major histocompatibility complex class I presentation of ovalbumin peptide 257-264 from exogenous sources: protein context influences the degree of TAP-independent presentation. Eur J Immunol 1996, 26:2790-2799.

26. Carreno $L J$, Bueno SM, Bull P, Nathenson SG, Kalergis AM: The half-life of the T-cell receptor/peptide-major histocompatibility complex interaction can modulate T-cell activation in response to bacterial challenge. Immunology 2007, 121:227-237.

27. Higgins DE, Shastri N, Portnoy DA: Delivery of protein to the cytosol of macrophages using Escherichia coli K-12. Mol Microbiol 1999, 31:1631-1641.

28. Pope C, Kim SK, Marzo A, Masopust D, Williams K, Jiang J, Shen H, Lefrancois L: Organ-specific regulation of the CD8 T cell response to Listeria monocytogenes infection. J Immunol 2001, 166:3402-3409.

29. Serre K, Mohr E, Toellner KM, Cunningham AF, Granjeaud S, Bird R, MacLennan IC: Molecular differences between the divergent responses of ovalbumin-specific CD4 T cells to alum-precipitated ovalbumin compared to ovalbumin expressed by Salmonella. Mol Immunol 2008, 45:3558-3566.

30. Barat S, Willer Y, Rizos K, Claudi B, Maze A, Schemmer AK, Kirchhoff D, Schmidt A, Burton N, Bumann D: Immunity to intracellular Salmonella depends on surface-associated antigens. PLoS Pathog 2012, 8:e1002966.

31. Maskell DJ, Sweeney KJ, O'Callaghan D, Hormaeche CE, Liew FY, Dougan G: Salmonella typhimurium aroA mutants as carriers of the Escherichia coli heat-labile enterotoxin $B$ subunit to the murine secretory and systemic immune systems. Microb Pathog 1987, 2:211-221.

32. Hone D, Attridge S, van den Bosch L, Hackett J: A chromosomal integration system for stabilization of heterologous gene in Salmonella based vaccine strains. Microb Pathog 1988, 5:407-418.

33. Froehlich BJ, Scott JR: A single-copy promoter-cloning vector for use in Escherichia coli. Gene 1991, 108:99-101.

34. McKelvie ND, Stratford R, Wu T, Bellaby T, Aldred E, Hughes NJ, Chatfield SN, Pickard D, Hale C, Dougan G, Khan SA: Expression of heterologous antigens in Salmonella Typhimurium vaccine vectors using the in vivo-inducible, SPI-2 promoter, ssaG. Vaccine 2004, 22:3243-3255.

35. Xu X, Husseiny Ml, Goldwich A, Hensel M: Efficacy of intracellular activated promoters for generation of Salmonella-based vaccines. Infect Immun 2010, 78:4828-4838.

36. Saxena M, Coloe PJ, Smooker PM: Influence of promoter, gene copy number, and preexisting immunity on humoral and cellular responses to a vectored antigen delivered by a Salmonella enterica vaccine. Clin Vaccine Immunol 2009, 16:78-87.

37. Bumann D: Regulated antigen expression in live recombinant Salmonella enterica serovar Typhimurium strongly affects colonization capabilities and specific CD4(+)-T-cell responses. Infect Immun 2001, 69:7493-7500.

38. Luria-Perez R, Cedillo-Barron L, Santos-Argumedo L, Ortiz-Navarrete VF, Ocana-Mondragon A, Gonzalez-Bonilla CR: A fusogenic peptide expressed on the surface of Salmonella enterica elicits CTL responses to a dengue virus epitope. Vaccine 2007, 25:5071-5085.

39. Jose J, Meyer TF: The autodisplay story, from discovery to biotechnical and biomedical applications. Microbiol Mol Biol Rev 2007, 71:600-619.

40. Loeffler DI, Schoen CU, Goebel W, Pilgrim S: Comparison of different live vaccine strategies in vivo for delivery of protein antigen or antigen-encoding DNA and mRNA by virulence-attenuated Listeria monocytogenes. Infect Immun 2006, 74:3946-3957.
41. Hegazy WA, Xu X, Metelitsa L, Hensel M: Evaluation of Salmonella enterica type III secretion system effector proteins as carriers for heterologous vaccine antigens. Infect Immun 2012, 80:1193-1202.

42. Hanahan D: Techniques for transformation of E. coli. In DNA cloning: a practical approach, Volume 1. Edited by Glover DM. Oxford, UK: IRL Press; 1985:109-135.

43. Yanisch-Perron C, Vieira J, Messing J: Improved M13 phage cloning vectors and host strains: nucleotide sequences of the M13mp18 and pUC19 vectors. Gene 1985, 33:103-119.

44. Hoiseth SK, Stocker BD: Aromatic-dependent Salmonella typhimurium are nonvirulent and effective as live vaccines. Nature 1981, 291:238-239.

45. Schödel F, Kelly SM, Peterson DL, Milich DR, Curtiss R 3rd: Hybrid hepatitis $B$ virus core-pre-S proteins synthesized in avirulent Salmonella typhimurium and Salmonella typhi for oral vaccination. Infect Immun 1994, 62:1669-1676.

46. Schifferli DM, Alrutz M: Permissive linker insertion sites in the outer membrane protein of 987P fimbriae of Escherichia coli. J Bacteriol 1994, 176:1099-1110.

47. Achtman M, Manning PA, Edelbluth C, Herrlich P: Export without proteolytic processing of inner and outer membrane proteins encoded by F sex factor Tra cistrons in Escherichia coli minicells. Proc Natl Acad Sci USA 1979, 76:4837-4841.

doi:10.1186/1475-2859-13-80

Cite this article as: Zhang et al:: Improved delivery of the OVA-CD4 peptide to T helper cells by polymeric surface display on Salmonella. Microbial Cell Factories 2014 13:80.

\section{Submit your next manuscript to BioMed Central and take full advantage of:}

- Convenient online submission

- Thorough peer review

- No space constraints or color figure charges

- Immediate publication on acceptance

- Inclusion in PubMed, CAS, Scopus and Google Scholar

- Research which is freely available for redistribution 